Check for updates

Cite this: RSC Adv., 2018, 8, 24094

\title{
Insight into the dispersion behavior of 1-butyl-3- methylimiazolium chloride confined in nanoscale pores of carbon materials $\uparrow$
}

\author{
Shuxia Di, (D) Yiqi Xu, Qunfeng Zhang, Xiaolong Xu, Yuanyuan Zhai, Bolin Wang, \\ Haihua He, Qingtao Wang, (D) Hao Xu, Yishu Jiang, Jia Zhao (DD and Xiaonian Li*
}

It is important to understand the behaviour of ionic liquids (ILS) in nanoscale pores, as application of supported ionic liquid phase (SILP) materials has attracted much attention. The main intention of this investigation is to study the dispersion performance of ionic liquid on activated carbons with different surface oxygen groups. Several active carbons with diverse oxygenated surface groups were obtained by oxidation and reduction methods. All samples were impregnated with $[\mathrm{Bmim}] \mathrm{Cl}$ at different loadings. The blocked porosity of ionic liquid on SILP materials mainly depends on the amount of ionic liquid and surface oxygen content. In addition, stability of the supported IL in water was tested by analyzing the leached amount of IL. We found that the supported IL is not easily leached from the interface of carbon with low amount of surface oxygen. This may be due to the high free $\pi$-electron density of oxygen-free supports, forming more $\mathrm{CH}-\pi$ bonds with $\mathrm{H}$ atoms at the $\mathrm{C} 2$ position of the acidic cation. In addition, we propose that dispersion of ionic liquid in the pores depends on the density of surface free $\pi$ electrons of carbon materials.

Received 9th April 2018

Accepted 15th June 2018

DOI: $10.1039 / c 8 r a 03033 b$

rsc.li/rsc-advances

\section{Introduction}

Ionic liquids possess an array of unique physicochemical properties, namely, negligible vapor pressure, relatively low viscosity, effective non-volatility, thermal stability, and relatively high ionic conductivity, ${ }^{1-3}$ and have strongly attracted scientific attention. They are considered as promising green solvents to replace conventional volatile organic compounds. A variety of new valuable applications of ILs have continuously been developed in some industries, such as gas separation, ${ }^{4-7}$ catalysts, ${ }^{8-10}$ extractants, ${ }^{11,12}$ materials synthesis, ${ }^{13,14}$ explosives and propellant fuels..$^{15}$ In some cases, it is necessary to immobilize ionic liquids on porous materials, which disperses the ionic liquids on the inter-surfaces of the porous structure and at the same time, allows the retention of high specific surface areas and structural properties. In particular to the catalysis field, such as in acetylene hydrochlorination reaction, ${ }^{16,17}$ the introduction of ionic liquids promotes both catalytic activity and stability of the catalysts. Therefore, it is of great value to investigate the dispersion behavior of ionic liquids as they play a key role in the SILC system, particularly in gas-solid reactions.

Industrial Catalysis Institute, Laboratory Breeding Base of Green Chemistry-Synthesis Technology, Zhejiang University of Technology, Hangzhou 310014, China. E-mail: jiazhao@zjut.edu.cn; xnli@zjut.edu.cn; Fax: $\quad+86$ 57188320002; $\quad$ Tel: $\quad+86$ 57188320002

$\dagger$ Electronic supplementary information (ESI) available. See DOI: $10.1039 / \mathrm{c} 8 \mathrm{ra0} 03033 \mathrm{~b}$
To our knowledge, ionic liquids can be easily supported on porous materials, forming functionalized materials with a "thin film", which has made SILP systems a topic of intensive scientific research. For example, Li et $a l .{ }^{16}$ proposed that the weak van der Waals interaction between imidazolium-based ionic liquid and single-walled carbon nanotubes (SWCNTs) dispersed the SWCNTs into the IL. Rodriguez et al. ${ }^{17}$ found that the IL tends to fill micropores first, then mesopores and finally macropores in SILP systems. Szesni et al. ${ }^{18}$ studied the distribution of IL immobilized onto silica gel using solid-state NMR characterization, presenting optimum ionic liquid loading. Stark et al. ${ }^{19}$ studied the solid-ionic liquid interfaces between IL and ordered or disordered mesoporous silica; they proposed that the formation of layer or droplet depended on the physicochemical properties of the ionic liquid. To the best of our knowledge, only the stability (immobilization and thermal stability) of immobilized ionic liquids has so far been reported, ${ }^{20}$ but almost no group has analyzed the relationship between IL and carbon materials in detail due to the complexity of surface chemicals and random porosity of the carbon materials. The relation of dispersion behavior of ionic liquid confined in nanoscale pores of carbon materials to the surface chemical properties is still an open question.

In the present study, a series of carbon materials with different surface chemical functional groups were investigated. Also, the dispersion behavior of ionic liquid supported on carbon materials was analyzed via a variety of characterization methods. We discovered that the surface free $\pi$-electron density 
of porous carbon materials not only enhanced the distribution of ionic liquid on the surface of porous carbons materials, but also contributed to immobilizing the ionic liquid on the support due to the formation of $\mathrm{CH}-\pi$ bond with $\mathrm{H}$ atom at the $\mathrm{C} 2$ position of the acidic cation.

\section{Experimental}

\subsection{Preparation of SILP materials}

Columnar activated carbon NORIT ROX 0.8 with diameter of $0.8 \mathrm{~mm}$ and length of $5 \mathrm{~mm}$ was chosen as the support, which contains almost no surface oxygen groups. In order to investigate effects of surface oxygen groups on distribution behavior of ionic liquid on the surface of activated carbon, the original carbon material was treated with concentrated nitric acid, followed by thermal treatment.

Specifically, the surface oxygen groups on activated carbon were introduced by dipping the activated carbon into concentrated nitric acid ( $65 \mathrm{wt} \%$ ) at room temperature for $12 \mathrm{~h}$. Then, the activated carbon was filtered and rinsed with deionized water until the $\mathrm{pH}$ value of the filtrate was about 7. Eventually, the treated activated carbon was dried at $120^{\circ} \mathrm{C}$ under vacuum for $12 \mathrm{~h}$ and labelled as AC-n. Next, the as-prepared AC-n was thermally treated at $500{ }^{\circ} \mathrm{C}$ or $900{ }^{\circ} \mathrm{C}$ for $4 \mathrm{~h}$ in $\mathrm{N}_{2}$ atmosphere, with a heating rate of $10^{\circ} \mathrm{C} \mathrm{min}^{-1}$, and the obtained samples were denoted as AC-n-N500 and AC-n-N900, respectively.

In this study, the SILP materials were prepared from the treated activated carbon by loading different amounts of 1butyl-3-methylimiazolium chloride $([\mathrm{Bmim}] \mathrm{Cl})$. The SILP materials with different IL loadings of $10 \mathrm{wt} \%, 20 \mathrm{wt} \%$ and $40 \mathrm{wt} \%$ were treated in vacuum environment, so that the entire impregnation solution could be adsorbed into the pores of the supports. Then, the materials were dried at $120{ }^{\circ} \mathrm{C}$ under vacuum for $12 \mathrm{~h}$ to remove water. The obtained SILP materials were labeled as AC-n- $10 \mathrm{wt} \%$, AC-n- $20 \mathrm{wt} \%$, AC-n- $40 \mathrm{wt} \%$, AC-nN500-10 wt \%, AC-n-N500-20 wt\%, AC-n-N500-40 wt\%, AC-nN900-10 wt\%, AC-n-N900-20 wt\%, and AC-n-N900-40 wt\%, according to preparation method.

\subsection{Characterization of SILP materials}

The types and amounts of oxygenated surface groups on activated carbon were determined by $\mathrm{CO}$ - and $\mathrm{CO}_{2}$-temperatureprogrammed desorption (TPD) using automated AMI-200 equipment (Altamira Instruments). The sample $(100 \mathrm{mg}$ ) was placed in a quartz tube and heated from $30{ }^{\circ} \mathrm{C}$ to $900{ }^{\circ} \mathrm{C}$ at a heating rate of $5^{\circ} \mathrm{C} \mathrm{min}{ }^{-1}$ under helium flow $\left(30 \mathrm{~mL} \mathrm{~min}^{-1}\right)$. $\mathrm{CO}$ and $\mathrm{CO}_{2}$ signals were monitored by a quadrupole mass spectrometer. TPD profiles of $\mathrm{CaC}_{2} \mathrm{O}_{4}$ were used to calibrate the quantification of $\mathrm{CO}$ and $\mathrm{CO}_{2}$ released from carbon materials.

$\mathrm{N}_{2}$ adsorption-desorption was employed to measure porous structure parameters of the supports and SILP materials on a Micromeritics ASAP 2020 instrument. Before measurements, all samples were degassed at $150{ }^{\circ} \mathrm{C}$ for $3 \mathrm{~h}$. The BET equation was used to calculate specific surface areas $\left(S_{\mathrm{BET}}\right)$; pore size distributions and pore volumes were determined by BJH model.

\section{Results and discussion}

\subsection{Carbon materials characterization}

In order to investigate the physical structures of activated carbon, the Brunauer-Emmett-Teller (BET) method was employed to clarify specific textural parameters of the asprepared materials. In addition, TPD experiments were carried out to characterize the surface chemistry of activated carbon materials.

3.1.1 Textural properties of AC. Fig. 1 shows nitrogen adsorption-desorption isotherms of the treated activated carbon materials (AC-n, AC-n-N500, AC-n-N900). The thermal treatment produced noticeable improvement in the adsorption capacity for both AC-n-N500 and AC-n-N900. Also, the adsorption capacity increased with the increase in the treatment temperature.

The textural parameters of the three samples were calculated from the nitrogen physisorption experiments and are summarized in Table 1. Results suggested that no significant differences in textural properties exist among the activated carbons that were thermally treated in inert atmosphere and under different temperatures. The specific surface area and pore volume for all the materials increased, which might be caused by the sharp decrease in oxygenated groups.

It has been reported in many studies that thermal treatments for activated carbon under inert atmosphere did not engender drastic changes to its textural properties, ${ }^{21,22}$ which conforms with the results summarized in Table 1.

3.1.2 Surface chemical properties. $\mathrm{CO}_{2}$ and $\mathrm{CO}$ desorption profiles (Fig. 2a and b, respectively) for the three activated carbon materials were obtained by TPD characterization. It is clear that the AC-n sample, treated with concentrated $\mathrm{HNO}_{3}$, contained the highest amount of surface oxygen groups compared to the other two samples with higher thermal treatment. The amounts of released $\mathrm{CO}_{2}$ and $\mathrm{CO}$ were assessed from the TPD profiles and the related data are collected in Table 2 together with the corresponding total amount of atomic oxygen (calculated from the amounts of $\mathrm{CO}_{2}$ and $\mathrm{CO}$ evolved). The

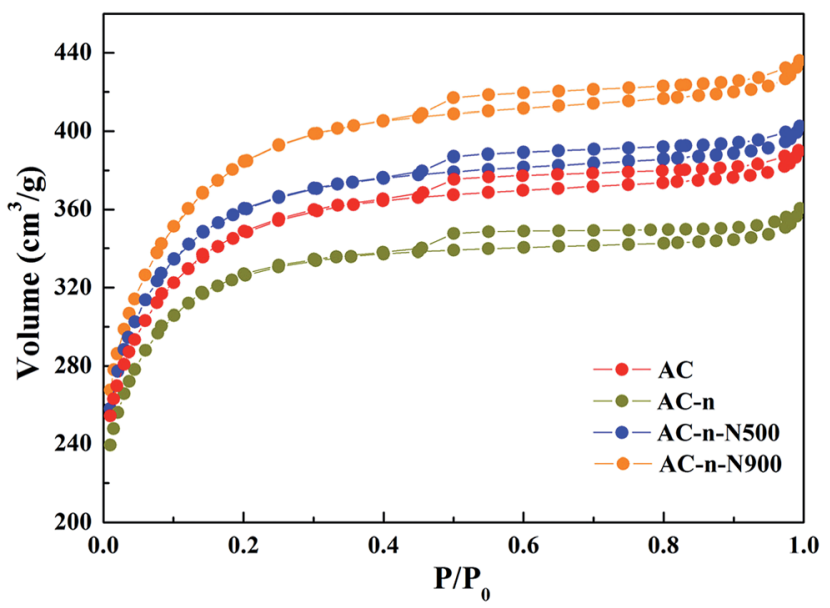

Fig. 1 Nitrogen adsorption-desorption isotherms of different carbon samples. 
Table 1 Textural properties of different activated carbons

\begin{tabular}{llllll}
\hline Sample & $S_{\text {BET }}\left(\mathrm{m}^{2} \mathrm{~g}^{-1}\right)$ & $D_{\text {pore }}(\mathrm{nm})$ & $V_{\text {pore }}\left(\mathrm{cm}^{3} \mathrm{~g}^{-1}\right)$ & $V_{\text {Microp. }}\left(\mathrm{cm}^{3} \mathrm{~g}^{-1}\right)$ & 0.3357 \\
\hline AC-n & 1012.82 & 2.20 & 0.5576 & 0.3440 & 0.2219 \\
AC-n-500 & 1126.22 & 2.21 & 0.6225 & 0.3146 & 0.2785 \\
AC-n-900 & 1216.95 & 2.22 & 0.6743 & 0.3597 \\
\hline
\end{tabular}
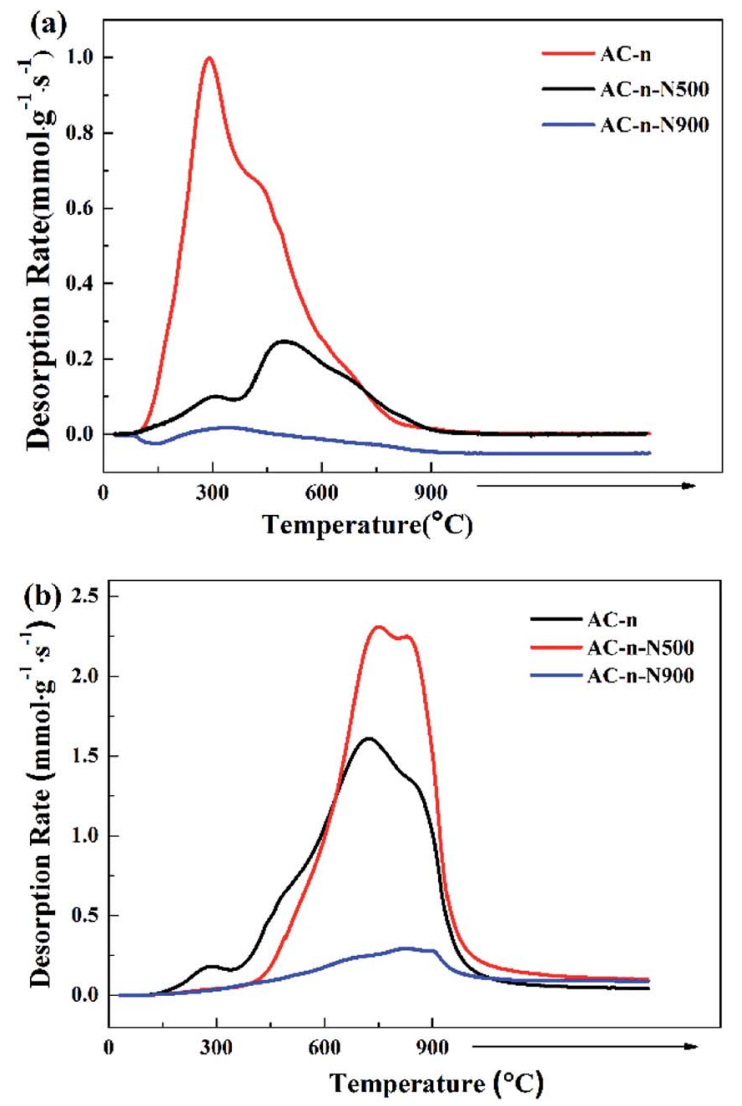

Fig. 2 TPD evolution profiles for different carbon samples: (a) $\mathrm{CO}_{2}$ and (b) CO.

different surface species leading to $\mathrm{CO}_{2}$ and $\mathrm{CO}$ evolution were obtained and are listed in the ESI (Tables S1 and S2 $\dagger$ ).

As expected, the data in Fig. 2 and Table 2 show that amount of surface oxygen groups in the AC-n samples decreased upon thermal treatment. However, thermal treatment at different temperatures led to different surface chemistries of the carbon materials. Treatment at $500{ }^{\circ} \mathrm{C}$ under nitrogen atmosphere resulted in the decomposition of carboxylic acid groups. Further, almost all of the surface oxygen groups decomposed at $900{ }^{\circ} \mathrm{C}$. The results of the TPD profiles were consistent with those obtained in a previous study. ${ }^{23}$

Table 2 Quantification of TPD profiles of treated activated carbons

\begin{tabular}{llll}
\hline Samples & $\mathrm{CO}_{2}\left(\mu \mathrm{mol} \mathrm{g}^{-1} \mathrm{~s}^{-1}\right)$ & $\mathrm{CO}\left(\mu \mathrm{mol} \mathrm{g}^{-1} \mathrm{~s}^{-1}\right)$ & $\mathrm{O} \mathrm{wt} \%$ \\
\hline AC-n & 301.3 & 593.3 & 1.9 \\
AC-n-N500 & 103.2 & 686.5 & 1.4 \\
AC-n-N900 & 0 & 155.2 & 0.2
\end{tabular}

In summary, the modified activated carbon differs widely in surface oxygen content and the nature of the surface oxygen groups from the untreated activated carbon. Therefore, when this series of samples were used as supports for SILP materials, the effects of surface oxygen chemistry on IL distribution and the interfacial effect of the SILP materials can be analyzed.

\subsection{SILP materials properties}

In SILP materials, it is likely that ionic liquids can be distributed on both external and internal surfaces of the supports. Therefore, it is relevant to investigate the effect of surface chemical properties of activated carbon on dispersion behavior of ionic liquid on SILP materials. In the present study, we analyzed the physical properties of SILP materials with different IL loadings, namely, surface area, pore volume, pore diameter distribution, and blocked porosity. Furthermore, we studied the stability of the SILP materials in water using weighing method.

3.2.1 Textural properties of SILP materials. In our study, we employed BET characterization to explore the distribution behavior of IL on the SILP materials. The textural parameters of these samples were calculated based on the nitrogen physisorption results, which are summarized in Table 3. As expected, impregnating activated carbon supports with increasing amounts of ionic liquid led to loss of pore volume and surface area, accompanied by an increase in mean pore diameter because IL first filled into the micropores, then the mesopores and finally the macropores. ${ }^{17}$ For these three SILP samples, there was a significant difference for the increasing trends of the mean pore diameter: AC-n increased from 2.20 to $5.97 \mathrm{~nm}$, AC-N-500 increased from 2.21 to $4.88 \mathrm{~nm}$, and AC-N-900 increased from 2.22 to $3.18 \mathrm{~nm}$. This should be due to the different surface chemistries of ACs and will be discussed in more depth in the later section. As shown in Fig. 3, when the IL loading was $10 \mathrm{wt} \%$ and $40 \mathrm{wt} \%$, the loss in BET surface area was similar for these three supports. However, when the IL loading was $20 \mathrm{wt} \%$, there was a significant difference in loss of BET surface area among these three samples (Fig. 3). This is accounted by the distribution behavior of IL on the surface of the support, which will be discussed in the next section.

3.2.2 Porosity of IL supported on carbon with different surface chemical properties. To obtain deeper insight into the distribution behavior of IL on the surface of the support, the related data were determined and collected in Table 4. Column 1 shows the total pore volume of the support and of the corresponding IL/AC sample. Column 2 shows the "non-accessible pore volume" calculated as the total pore volume of the support minus total pore volume of the IL/AC sample. Column 3 shows the volume of the loaded IL. Column 4 shows the 
Table 3 Different SILP materials' characterization

\begin{tabular}{lrllll}
\hline Sample & $S_{\text {BET }}\left(\mathrm{m}^{2} \mathrm{~g}^{-1}\right)$ & $D_{\text {pore }}(\mathrm{nm})$ & $V_{\text {pore }}\left(\mathrm{cm}^{3} \mathrm{~g}^{-1}\right)$ & $V_{\text {Microp. }}\left(\mathrm{cm}^{3} \mathrm{~g}^{-1}\right)$ & $V_{\text {Mecrop. }}\left(\mathrm{cm}^{3} \mathrm{~g}^{-1}\right)$ \\
\hline AC-n & 1012.82 & 2.20 & 0.5576 & 0.3357 & 0.2219 \\
AC-n-10\% IL & 863.60 & 2.29 & 0.4530 & 0.2184 & 0.2346 \\
AC-n-20\% IL & 297.70 & 2.42 & 0.1794 & 0.0260 & 0.1534 \\
AC-n-40\% IL & 17.07 & 5.97 & 0.0255 & 0.0017 & 0.0238 \\
AC-n-500 & 1126.22 & 2.21 & 0.6225 & 0.3440 & 0.2785 \\
AC-n-500-10\% IL & 975.95 & 2.24 & 0.5184 & 0.2577 & 0.2607 \\
AC-n-500-20\% IL & 521.88 & 2.41 & 0.3145 & 0.1015 & 0.2130 \\
AC-n-500-40\% IL & 25.32 & 4.88 & 0.0308 & 0.0025 & 0.0283 \\
AC-n-900 & 1216.95 & 2.22 & 0.6743 & 0.3146 & 0.3597 \\
AC-n-900-10\% IL & 1094.67 & 2.22 & 0.5697 & 0.2849 & 0.2848 \\
AC-n-900-20\% IL & 724.59 & 2.24 & 0.4058 & 0.1351 & 0.2707 \\
AC-n-900-40\% IL & 89.77 & 3.18 & 0.0715 & 0.0052 &
\end{tabular}

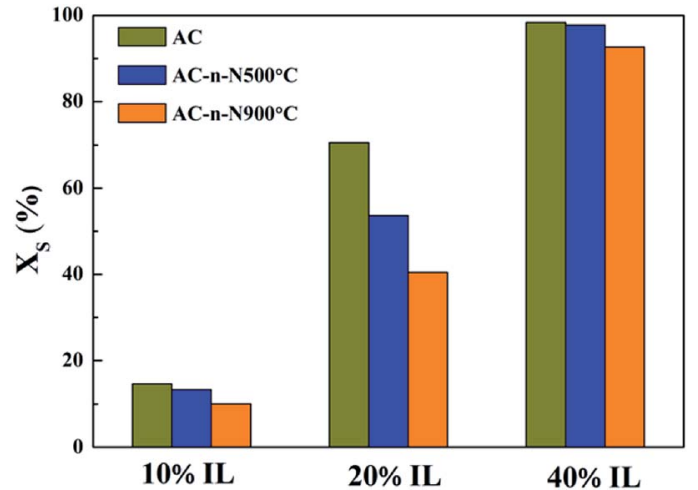

Fig. 3 Decrease in BET surface area of SILP materials with different supports on increasing the loading of IL, as obtained from adsorptiondesorption isotherms of $N_{2} ; S=\left(S_{0}-S_{i}\right) / S_{0} ; S_{0}$ : BET surface area of original supports; $S_{i}$ : BET surface area of SILP samples.

"blocked porosity" calculated as follows: the "non-accessible pore volume" (column 2) minus the volume of loaded IL in each IL/AC sample (column 3). The blocked porosity is expressed as an absolute value in $\mathrm{cm}^{3} \mathrm{~g}^{-1}$ and as $\%$ of the total pore volume of the support (in parentheses). It has been assumed that the IL volume does not change under the conditions of $\mathrm{N}_{2}$ adsorption $\left(-196^{\circ} \mathrm{C}\right)$ for this calculation.

Table 4 shows that the pores of all samples were blocked to a certain extent, which has been previously reported. ${ }^{20}$ This is because the ionic liquid did not exist as a uniform or consecutive film. For the three samples, with the increase in IL loading, the blocked porosity of AC-n first rose and then dropped, while that of both AC-n-N500 and AC-n-N500 increased monotonically. When IL loading is $10 \mathrm{wt} \%$, the blocked porosity for all samples was relatively low and almost the same as interface interaction between IL and active carbon played a predominant role, leading to well distributed IL on different active carbon materials. However, when IL loading reached $20 \mathrm{wt} \%$, the blocked porosity of the three samples exhibited significant differences. The blocked porosity of AC-n was $34.61 \%$, while that of AC-n-500 was only $11.91 \%$. These results suggested that larger interaction exists between IL and the surface of active carbon calcinated at high temperature,

Table 4 Analysis of the blocked porosity degree for SILP materials with different surface oxygen content ${ }^{a}$

\begin{tabular}{|c|c|c|c|c|}
\hline Sample & $\begin{array}{l}\text { Total pore } \\
\text { volume }\left(\mathrm{cm}^{3} \mathrm{~g}^{-1}\right)\end{array}$ & $\begin{array}{l}\text { Not accessible } \\
\text { pore volume }\left(\mathrm{cm}^{3} \mathrm{~g}^{-1}\right)\end{array}$ & $\begin{array}{l}\text { Loaded IL } \\
\text { volume }\left(\mathrm{cm}^{3} \mathrm{~g}^{-1}\right)\end{array}$ & $\begin{array}{l}\text { Blocked porosity } \\
\left(\mathrm{cm}^{3} \mathrm{~g}^{-1}\right)\end{array}$ \\
\hline AC-n & 0.5576 & - & - & - \\
\hline AC-n-10\% IL & 0.4530 & 0.1046 & 0.0926 & $0.0120(2.15 \%)$ \\
\hline AC-n-20\% IL & 0.1794 & 0.3782 & 0.1852 & $0.1930(34.61 \%)$ \\
\hline AC-n-N500-10\% IL & 0.5184 & 0.1041 & 0.0926 & $0.0155(1.85 \%)$ \\
\hline AC-n-N500-20\% IL & 0.3145 & 0.3080 & 0.1852 & $0.1228(19.73 \%)$ \\
\hline AC-n-N500-40\% IL & 0.0308 & 0.5917 & 0.3704 & $0.2213(35.55 \%)$ \\
\hline AC-n-N900 & 0.6743 & - & - & - \\
\hline AC-n-N900-10\% IL & 0.5697 & 0.1046 & 0.0926 & $0.0120(1.78 \%)$ \\
\hline AC-n-N900-20\% IL & 0.4058 & 0.2655 & 0.1852 & $0.0803(11.91 \%)$ \\
\hline
\end{tabular}

${ }^{a} 1$ - Total pore volume, determined by amount adsorbed at $P / P_{0}=0.99$ in $\mathrm{N}_{2}$ adsorption isotherm at $-196{ }^{\circ} \mathrm{C} .2-$ Pore volume not accessible to $\mathrm{N}_{2}$ determined as total pore volume of support AC minus total pore volume of SILP samples. 3 - Amount of IL in SILP samples (loaded IL volume calculated with $\left.\rho=1.08 \mathrm{~g} \mathrm{~cm}^{-3}\right) .4$ - Calculated by difference of columns 2 and 3 . In parentheses: percentage of support total pore volume marked as XV, that is (blocked porosity $\left(\mathrm{cm}^{3} \mathrm{~g}^{-1}\right) /$ support total pore volume $\left.\left(\mathrm{cm}^{3} \mathrm{~g}^{-1}\right)\right) \times 100$. 
promoting the dispersion of IL over the active carbon and thus decreasing the blocked porosity. Therefore, the variation in blocked porosity is mainly related to the loading amounts of IL and surface chemistry of the supports.

To investigate the influence of the surface chemistry of activated carbon on blocked porosity, the relationship between surface oxygen content of carbon materials and decrease in their pore volume and surface area was obtained. As shown in Fig. 4a and b, the BET surface area and the blocked porosity of pore volume within SILP materials were drastically decreased due to the reduction of surface oxygen content. Hence, we considered that the distribution performance of IL was related to the surface oxygen content of carbon materials.

3.2.3 Stability of IL supported on carbon with different surface chemical properties in water. In a previous study, Linares-Solano et $a .^{20}$ investigated the stability of IL/AC samples in water. They proposed that the hydrophilic ionic liquid supported on AC could be leached by deionized water. ${ }^{24,25}$ In this study, we adopted the same approach to wash the physisorbed ionic liquid from the activated carbon supports. Therefore, the quantification of covalently anchored ionic liquid could be achieved. The specific method is as follows: the as-prepared sample with $10 \mathrm{wt} \%$ IL loading was first dispersed in water for 30 minutes, and then centrifuged and filtered. This step was repeated until the filtrate contained no chloride ions, which was confirmed by titration with $\mathrm{AgNO}_{3}$ solution. Then, the washed samples were weighed after drying at $120{ }^{\circ} \mathrm{C}$ under vacuum for $12 \mathrm{~h}$.
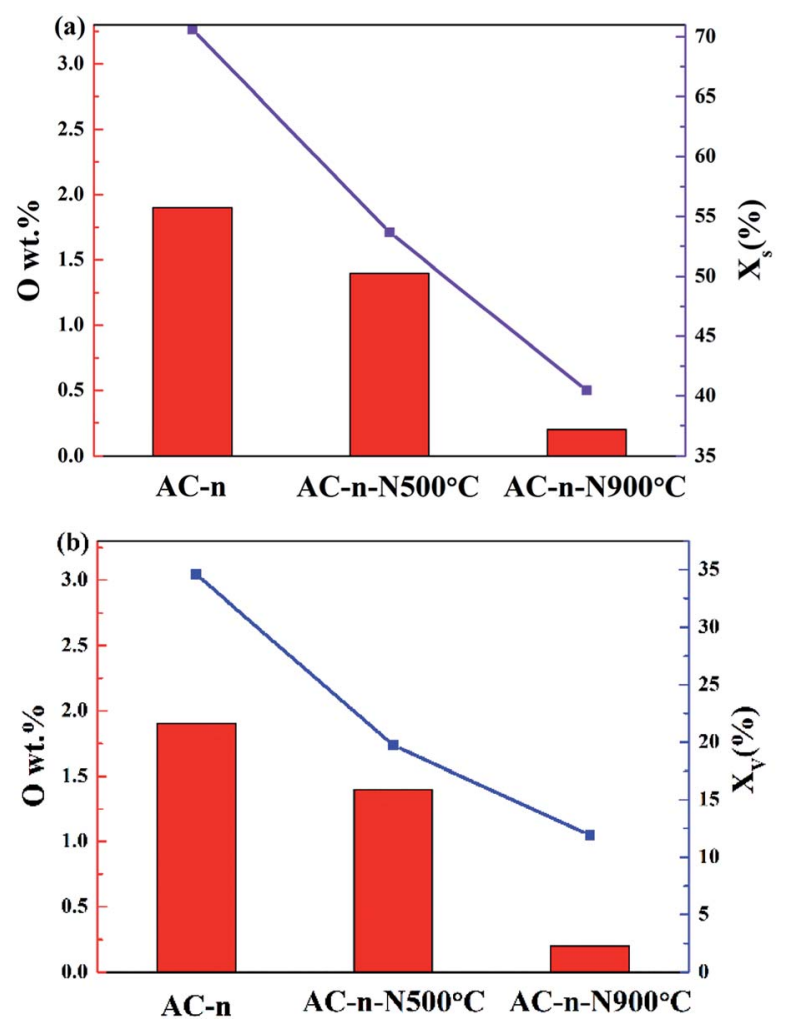

Fig. 4 (a) Decrease in BET surface area and (b) blocked porosity of pore volume in SILP materials related to surface oxygen content of carbon materials.
Fig. 5 shows the colour change of the filtrate in $\mathrm{AgNO}_{3}$ solution with the increase in washing. As the washing cycles increased, the colour of filtrate in $\mathrm{AgNO}_{3}$ solution changed from ivory-white to watery-white, suggesting that less ionic liquid was being washed off. Furthermore, we employed the Mohr method (GB11896-89) to analyze the amount of chloridion in the filtrate. Table 5 showed that concentration of chloridion in the filtrate decreased with the washing cycle. When the washing cycle increased to four times, for these three samples, concentration of chloridion in the filtrate was only $100 \mathrm{ppb}$. There are two reasons accounting for this finding: the first is that most of the ionic liquid was washed away by the water; the second is that part of the ionic liquid was difficult to wash off because of strong interactions between ionic liquid and active carbon.

Table 6 suggests that the ionic liquid loaded on the carbon materials was not washed completely. Furthermore, the residual quantity of the ionic liquid on active carbon varied among different treatment methods.

After four washing cycles, the actual immobilization values of AC-n, AC-n-500 and AC-n-900 with 10 wt\% IL loading were $1.6 \%, 2.4 \%$ and $3.6 \%$, respectively. A number of studies have reported that surface polarity of AC supports was enhanced by the increase in surface oxygen content, making them adsorb polar molecules more easily. However, this theory does not explain the results obtained in this experiment as the actual immobilization amount increased with the reduced surface oxygen content of the AC support (Tables 2 and 6).

Therefore, three aspects must be considered to explain these results. (i) The first aspect is the phase state of immobilized ionic liquid. For example, Hu et al. ${ }^{26}$ proposed that the coexistence of liquid and solid phases for ionic liquid was intuitively observed on the surface of mica using atomic force microscopy (AFM). Also, the drop-on-the-layer phenomenon, proposed by this group, was believed to be a special microscopic property of liquids when contacted with a solid surface. Furthermore, the drop layer had the natural property of the bulk phase. In addition, Hardacre et $a .^{27}$ proposed multilayer stacking of ILs on the surface of $S_{\mathrm{i}}$ (111) wafers using X-ray diffraction. Therefore, in our study, liquid and solid phases of the ionic liquid may coexist on the carbon materials, from which the liquid phase is easily leached, but the solid phase is hardly leached. (ii) The $\mathrm{H}$ atom in the $\mathrm{C} 2$ position of imidazolium cation of the ionic liquid possesses high acidity to easily form $\mathrm{CH}-\pi$ hydrogen bond with free $\pi$-electrons. For example, Sheng Su et al. $^{28}$ proposed a strong interaction between IL and CNT from thermogravimetric characterization and further verified the formation of $\mathrm{CH}-\pi$ hydrogen bond ${ }^{29-31}$ between IL and CNT using X-ray diffraction and FTIR spectra. The properties of hydrogen bonds have been studied by a number of researchers. ${ }^{32-34}$ In nanoscience, the $\pi-\mathrm{H}$ hydrogen bonds are currently characterized by their weak interaction strength, where the contribution of dispersion energy dominates upon the formation and stabilization of the analysed system. ${ }^{35}$ (iii) Carbon materials having $\mathrm{C}=\mathrm{C}$ bonds, particularly oxygenfree carbon materials can release some amount of free $\pi$ electrons. ${ }^{36-38}$

As shown in Fig. $\mathrm{S} 1, \dagger$ it reported that the content of $\mathrm{C}=\mathrm{C}$ bond increased through thermal treatment and hence, $\mathrm{C}=\mathrm{C}$ 


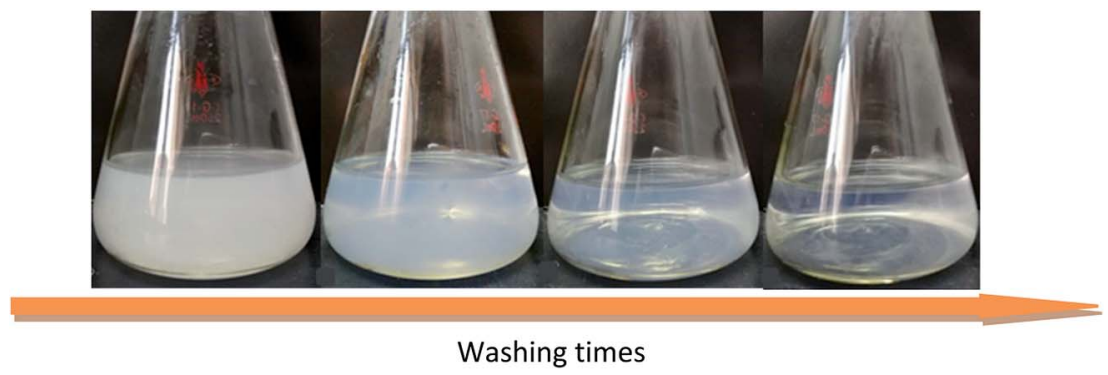

Fig. 5 Color change of filtrate in $\mathrm{AgNO}_{3}$ solution with four washes.

Table 5 Concentration of chloridion in filtrate after each wash

\begin{tabular}{lllll}
\hline Sample & First wash $\left(C_{\mathrm{Cl}^{-}}: \mathrm{mol} \mathrm{L}^{-1}\right)$ & Second wash $\left(C_{\mathrm{Cl}^{-}}: \mathrm{mol} \mathrm{L}^{-1}\right)$ & Third wash $\left(C_{\mathrm{Cl}^{-}}: \mathrm{mol} \mathrm{L}^{-1}\right)$ & Fourth wash $\left(C_{\mathrm{Cl}^{-}}: \mathrm{mol} \mathrm{L}^{-1}\right)$ \\
\hline AC-n & $5.19 \times 10^{-3}$ & $6.97 \times 10^{-4}$ & $5.81 \times 10^{-5}$ & $6.12 \times 10^{-7}$ \\
AC-n-N500 & $3.52 \times 10^{-3}$ & $2.10 \times 10^{-4}$ & $2.07 \times 10^{-5}$ & $6.08 \times 10^{-7}$ \\
AC-n-N900 & $2.13 \times 10^{-3}$ & $1.09 \times 10^{-4}$ & $2.08 \times 10^{-5}$ & $6.01 \times 10^{-7}$
\end{tabular}

Table 6 Analysis results of different SILP materials after washing

\begin{tabular}{llll}
\hline Sample & $\begin{array}{l}\text { Theoretical } \\
\text { value/g }\end{array}$ & $\begin{array}{l}\text { Actual } \\
\text { value/g }\end{array}$ & $\begin{array}{l}\text { Actual } \\
\text { immobilization/\% }\end{array}$ \\
\hline AC-n & 5.5 & 5.08 & 1.6 \\
AC-n-500 & 5.5 & 5.12 & 2.4 \\
AC-n-900 & 5.5 & 5.18 & 3.6
\end{tabular}

bonds can release more free $\pi$-electrons. ${ }^{39,40}$ Therefore, we suggest a reasonable explanation for the immobilization behaviour of ionic liquid. The interaction between IL and the surface of active carbon, viz., the $\mathrm{CH}-\pi$ hydrogen bond, has a crucial effect on the stability of IL supported on carbon materials. As shown in Fig. 6, there are two phases of IL on the activated carbon. One is called the "solid-phase ionic liquid", where IL is well-distributed on the support through the formation of $\mathrm{CH}-\pi$ hydrogen bonds between IL and the surface of AC. The other phase of ionic liquid, apart from the disordered molecular arrangement on the surface of active carbon, is called the "liquid-phase ionic liquid" and its physicochemical

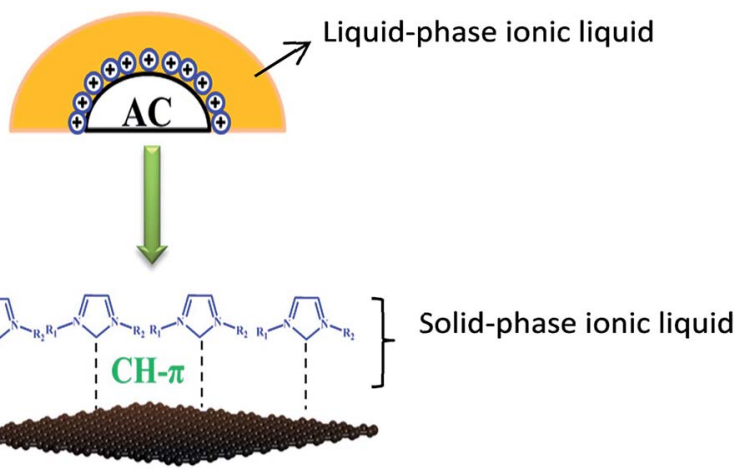

Fig. 6 Schematic of interaction between ionic liquid and carbon surface (orange: liquid-phase ionic liquid; blue: solid-phase ionic liquid). property is similar to that of the ionic liquid in bulk phase, which is easily washed off by water. In this experiment, a large number of oxygen functional groups were introduced by treating the activated carbon with concentrated nitric acid and thus, the density of free $\pi$-electrons decreased and consequently, the intensity of $\mathrm{CH}-\pi$ hydrogen bonds between IL and the surface of AC was weakened. As a result, the ionic liquid became unstable and could easily be washed from the support. However, when active carbons were calcined under inert gas, the surface oxygen decomposed and desorbed in the form of carbon monoxide and carbon dioxide, thus increasing the density of free $\pi$-electrons. In this case, the interaction intensity between IL and surface of active carbon enhanced and hence, the IL was more difficult to wash off.

Moreover, the disparity of IL supported on activated carbon depended on the density of the surface free $\pi$-electrons due to the formation of $\mathrm{CH}-\pi$ bonds between the surface of AC and IL. The blocked porosity could not be easily formed because of the surface chemical effects with low IL loading. Comparing the data for the three SILP samples with $20 \mathrm{wt} \%$ IL loading, we found that supporting IL on AC-n resulted in the highest blocked porosity because of the lower density of free $\pi$-electrons on the support, causing disorder of IL at the interface. On the contrary, the distribution of IL was more ordered on the supports with high temperature treatment for the formation of $\mathrm{CH}-\pi$ bonds between the surface of AC and IL.

\section{Conclusion}

The distribution behavior of ionic liquid supported on porous carbon materials was investigated using a series of carbon supports with a variety of textural, chemical and morphological properties. We found that the blocked porosity of the ionic liquid on SILP materials was not only related to the amount of ionic liquid, but also to the surface oxygen content. The blocked porosity decreased for carbon materials with low surface oxygen 
content. Furthermore, the stability of the supported IL was tested in water until the IL was not leached. We discovered that the supported IL was not easily washed from the activated carbon with low concentration of surface oxygen groups because the oxygen-free supports have a high density of free $\pi$ electrons and the $\mathrm{H}$ atom on the $\mathrm{C} 2$ position of the acidic cation in the IL structure forms a $\mathrm{CH}-\pi$ bond with the surface $\pi$ electrons on carbon materials. In addition, we proposed that the distribution of ionic liquid supported on thermally treated activated carbon could be enhanced by increasing the density of surface free $\pi$-electrons of carbon materials. While investigating IL loading, the supported IL led to some blocked porosity for three different carbons. However, the distribution of IL on the surface of activated carbon supports with high temperature treatment is much more ordered than that on the merely oxidized activated carbon support. These results showed that the formation of $\mathrm{CH}-\pi$ hydrogen bonds between the ionic liquids with imidazole ring and carbon materials with high free $\pi$-electrons can enhance the dispersion of ionic liquid on the surface of the support.

\section{Conflicts of interest}

There are no conflicts to declare.

\section{Acknowledgements}

Financial support by the National Natural Science Foundation of China (NSFC; Grant 21606199, 21476207) is gratefully acknowledged.

\section{Notes and references}

1 K. R. Seddon, J. Chem. Technol. Biotechnol., 1997, 68, 351356.

2 P. Wasserscheid and W. Keim, Angew. Chem., Int. Ed., 2000, 39, 3772-3789.

3 E. D. Bates, R. D. Mayton, I. Ntai and J. H. Davis, J. Am. Chem. Soc., 2002, 124, 926-927.

4 M. E. Bluhm, M. G. Bradley, R. Butterick, U. Kusari and L. G. Sneddon, J. Am. Chem. Soc., 2006, 128, 7748-7749.

5 G. B. Damas, A. B. A. Dias and L. T. Costa, J. Phys. Chem. B, 2014, 118, 9046-9064.

6 K. B. Sezginel, S. Keskin and A. Uzun, Langmuir, 2016, 32, 1139-1147.

7 Y. F. Chen, Y. Y. Zhang, S. J. Yuan, X. Y. Ji, C. Liu, Z. H. Yang and X. H. Lu, J. Chem. Eng. Data, 2016, 61, 3428-3437.

8 Q. H. Zhang, S. G. Zhang and Y. Q. Deng, Green Chem., 2011, 13, 2619-2637.

9 F. Jutz, J. M. Andanson and A. Baiker, Chem. Rev., 2011, 111, 322-353.

10 P. Zhang, T. B. Wu and B. X. Han, Adv. Mater., 2014, 26, 6810-6827.

11 E. S. Farrell and G. E. Pacey, Anal. Chem., 2000, 72, 18191822.

12 X. Q. Sun, H. M. Luo and S. Dai, Chem. Rev., 2012, 112, 21002128.
13 E. R. Cooper, C. D. Andrews, P. S. Wheatley, P. B. Webb, P. Wormald and R. E. Morris, Nature, 2004, 430, 1012-1016.

14 B. K. Price, J. L. Hudson and J. M. Tour, J. Am. Chem. Soc., 2005, 127, 14867-14870.

15 Q. H. Zhang and J. M. Shreeve, Chem. Rev., 2014, 114, 1052710574.

16 J. Y. Wang, H. B. Chu and Y. Li, ACS Nano, 2008, 2, 25402546.

17 J. Lemus, J. Palomar, M. A. Gilarranz and J. J. Rodriguez, Adsorption, 2011, 17, 561-571.

18 M. Haumann, A. Schönweiz, H. Breitzke, G. Buntkowsky, S. Werner and N. Szesni, Chem. Eng. Technol., 2012, 35, 1421-1426.

19 M. T. Heinze, J. C. Zill, J. Matysik, W. D. Einicke, R. Glaser and A. Stark, Phys. Chem. Chem. Phys., 2014, 16, 2435924372.

20 M. Rufete-Beneite, M. C. Roman-Martinez and A. LinaresSolano, Carbon, 2014, 77, 947-957.

21 E. G. Rodrigues, M. F. R. Pereira, X. W. Chen, J. J. Delgado and J. J. M. Orfao, J. Catal., 2011, 281, 119-127.

22 J. A. Menendez, J. Phillips, B. Xia and L. R. Radovic, Langmuir, 1996, 12, 4404-4410.

23 J. H. Xu, J. Zhao, J. T. Xu, T. T. Zhang, X. N. Li, X. X. Di, J. Ni, J. G. Wang and J. Cen, Ind. Eng. Chem. Res., 2014, 53, 1427214281.

24 J. G. Huddleston, A. E. Visser, W. M. Reichert, H. D. Willauer, G. A. Broker and R. D. Rogers, Green Chem., 2001, 3, 156-164.

25 Z. J. Chen, Z. P. Li, X. Y. Ma, P. F. Long, Y. Zhou, L. Xu and S. G. Zhang, Green Chem., 2017, 19, 1303-1307.

26 Y. Liu, Y. Zhang, G. Wu and J. Hu, J. Am. Chem. Soc., 2006, 128, 7456-7457.

27 J. C. Adrian, H. Christopher and D. H. John, Mol. Phys., 2001, 99, 795-800.

28 Y. X. Ding and D. S. Su, ChemSuschem, 2014, 7, 1542-1546.

29 S. J. Grabowski, Chem. Rev., 2011, 111, 2597-2625.

30 D. A. Rodham, S. Suzuki, R. D. Suenram, F. J. Lovas, S. Dasgupta, W. A. Goddard and G. A. Blake, Nature, 1993, 362, 735-737.

31 S. Tsuzuki, K. Honda, T. Uchimaru, M. Mikami and K. Tanabe, J. Am. Chem. Soc., 2000, 122, 11450-11458.

32 P. Tarakeshwar, H. S. Choi and K. S. Kim, J. Am. Chem. Soc., 2001, 123, 3323-3331.

33 S. Vaupel, B. Brutschy, P. Tarakeshwar and K. S. Kim, J. Am. Chem. Soc., 2006, 128, 5416-5426.

34 D. Kim, P. Tarakeshwar and K. S. Kim, J. Phys. Chem. A, 2004, 108, 1250-1258.

35 E. C. Lee, D. Kim, P. Jurečka, P. Tarakeshwar, P. Hobza and K. S. Kim, J. Phys. Chem. A, 2007, 111, 3446-3457.

36 D. Sebastián, Carbon, 2010, 48, 4421-4431.

37 Z. Hashisho, M. J. Rood, S. Barot and J. Bernhard, Carbon, 2009, 47, 1814-1823.

38 Z. Tabti and R. Ruiz-Rosas, ACS Appl. Mater. Interfaces, 2014, 6, 11682-11691.

39 M. Baranac-Stojanović, A. Koch and E. Kleinpeter, Chemistry, 2012, 18, 370.

40 S. Sarkar, S. Niyogi and E. Bekyarova, Chem. Sci., 2011, 2, 1326-1333. 